PROCEEDINGS OF THE

AMERICAN MATHEMATICAL SOCIETY

Volume 128, Number 7 , Pages 2181-2186

S 0002-9939(99)05240-5

Article electronically published on November 29, 1999

\title{
HIGHER ORDER SYMMETRIC SPACES AND THE ROOTS OF THE IDENTITY IN A LIE GROUP
}

\author{
CECÍLIA FERREIRA AND ARMANDO MACHADO
}

(Communicated by Roe Goodman)

\begin{abstract}
Let $r_{k}(G)$ denote the set of all $k$-roots of the identity in a Lie group $G$. We show that $r_{k}(G)$ is always an embedded submanifold of $G$, having the conjugacy classes of its elements as open submanifolds. These conjugacy classes are examples of $k$-symmetric spaces and we show, more generally, that every $k$-symmetric space of a Lie group $G$ is a covering manifold of an embedded submanifold $O r b$ of $G$. We compute also the Hessian of the inclusions of $r_{k}(G)$ and $O r b$ into $G$, relative to the natural connection on the domain and to the symmetric connection on $G$.
\end{abstract}

\section{INTRODUCTION}

There are several examples of homogeneous manifolds of a Lie group $G$ that can be realized equivariantly as connected components of the set $r_{k}(G)$ of all $k$-roots of the identity $e$ of $G$, with $G$ acting by conjugation, in particular as conjugation classes of elements of $G$ : If $E$ is a Hermitian vector space, the Grassmann manifold $G r_{p}(E)$, whose elements are the $p$-dimensional vector subspaces, can be realized as a connected component of $r_{2}(U(E))$, as observed by Uhlenbeck [6], and, in an analogous way, if $E$ is a Euclidean space, $G r_{p}(E)$ admits a connected component of $r_{2}(O(E))$ as an equivariant model; More generally, if $E$ is a Hermitian space, the connected components of $r_{k}(U(E))$ are models of the flag manifolds $\mathcal{G}_{p_{1}, \ldots, p_{k}}(E)$, whose elements are the systems $\left(F_{1}, \ldots, F_{k}\right)$ of mutually orthogonal subspaces, with dimensions $p_{1}, \ldots, p_{k}$, whose direct sum is $E$ [3]; If $E$ is a Euclidean space, the connected components of $r_{3}(O(E))$ are models of the manifolds $\mathcal{F}_{p}(E)$, whose elements are the $p$-dimensional partially complex structures, i.e. the couples $(F, J)$, where $J$ is a compatible complex structure on the $2 p$-dimensional real subspace $F \subset E$ [4], with the exception of the extreme case $\operatorname{dim}(E)=2 p$, where $\mathcal{F}_{p}(E)$ is the union of two connected components.

With the previous examples in mind, we prove that, for a general Lie group $G$, $r_{k}(G)$ is always a submanifold of $G$, in general with variable dimension, having the conjugation classes of its elements as open submanifolds (we will use always the word "submanifold" with the meaning "embedded submanifold").

Received by the editors April 24, 1998 and, in revised form, August 24, 1998.

1991 Mathematics Subject Classification. Primary 22E15; Secondary 53C30, 53C35.

Key words and phrases. Lie group, orbit, $k$-symmetric space, $k$-root of the identity.

This work was supported by FCT, PRAXIS XXI, FEDER and project PRAXIS/2/ 2.1/MAT/125/94. 
The conjugation classes of elements of $r_{k}(G)$ are examples of $k$-symmetric manifolds; they are of the form $G / G^{\tau}$, where $G^{\tau}$ is the fixed point subgroup of a smooth automorphism $\tau: G \rightarrow G$, satisfying $\tau^{k}=I d_{G}$. The structure and classification of $k$-symmetric manifolds have been extensively studied in [5] and it has been known for a long time (cf. 2]) that every 2-symmetric manifold of the form $G / G^{\tau}$ can be one-to-one immersed into the Lie group $G$, by associating $g \tau\left(g^{-1}\right)$ with the class of an element $g \in G$, a fact that can be generalized trivially to $k$-symmetric spaces. We will prove, more precisely, that this one-to-one immersion is always an embedding, and hence that the $k$-symmetric space $G / G^{\tau}$ admits a model $O r b$ that is a submanifold of $G$. This fact was established in [1, for the special case where $G$ is compact, where the name "Cartan embedding" is used (in fact, Burstall uses the inverse $\tau(g) g^{-1}$ instead of $g \tau\left(g^{-1}\right)$ but this makes no essential difference). Of course, for general $k$-symmetric spaces, those of the form $G / H$, with $H$ an open subgroup of $G^{\tau}$, all we can guarantee is that they are covering manifolds of the manifold Orb.

Every $k$-symmetric space is reductive in a canonical way and, as such, it has a canonical connection, and it is known 2] that, for $k=2$, its embedding into the Lie group $G$, considered with its symmetric connection, is totally geodesic. This fact led us to compute, for general $k$, the Hessian of the inclusions of $r_{k}(G)$ and Orb into $G$.

In the next section we will prove a basic lemma that guarantees that, under certain conditions, the image of a smooth map is a smooth manifold. This lemma will be used in section 3 in order to prove that Orb is a submanifold but it is possible that it may present some independent interest.

\section{A BASIC LEMMA}

Lemma 1. Let $X, Y$ and $Z$ be manifolds and $f: X \rightarrow Y$ and $\varphi, \psi: Y \rightarrow Z$ be smooth maps such that $\varphi \circ f=\psi \circ f$. Let $x_{0} \in X$ be such that every vector $v \in T_{f\left(x_{0}\right)}(Y)$ verifying the condition $D \varphi_{f\left(x_{0}\right)}(v)=D \psi_{f\left(x_{0}\right)}(v)$ is in the image of $D f_{x_{0}}: T_{x_{0}}(X) \rightarrow T_{f\left(x_{0}\right)}(Y)$. Then the set $B=\{y \in Y \mid \varphi(y)=\psi(y)\}$ is a submanifold of $Y$ at $f\left(x_{0}\right)$, with the image of $D f_{x_{0}}$ as tangent space, and $f(X)$ is a neighborhood of $f\left(x_{0}\right)$ in $B$; in particular $f(X)$ is also a submanifold of $Y$ at $f\left(x_{0}\right)$, with the same tangent space.

Proof. The question being local, we may assume that $X, Y$ and $Z$ are open sets in finite dimensional spaces $E, F$ and $G$ and that $x_{0}=0$ and $f\left(x_{0}\right)=0$. Let us fix norms in these vector spaces, let $H \subset F$ be a direct sum complement of the vector subspace $D f_{0}(E)$ and let $g=\psi-\varphi: Y \rightarrow G$. The fact that the linear map $D g_{0}: F \rightarrow G$ is one-to-one in $H$ allows us to consider $\delta>0$ such that, for each $w \in H,\left\|D g_{0}(w)\right\| \geq \delta\|w\|$ (if $H$ is not trivial, let $\delta$ be the minimum of $\left\|D g_{0}(w)\right\|$, for $w \in H$ with $\|w\|=1$ ). Let $\varepsilon>0$ be such that, for each $y \in F$ with $\|y\|<\varepsilon$, we have $\left\|D g_{y}-D g_{0}\right\| \leq \delta / 2$. By the mean value theorem, if $\left\|y^{\prime}\right\|<\varepsilon$ and $\left\|y^{\prime \prime}\right\|<\varepsilon$, then $\left\|g\left(y^{\prime}\right)-g\left(y^{\prime \prime}\right)-D g_{0}\left(y^{\prime}-y^{\prime \prime}\right)\right\| \leq \delta / 2\left\|y^{\prime}-y^{\prime \prime}\right\|$. Let $U \subset X$ and $W \subset H$ be open sets, with $0 \in U$ and $0 \in W$, such that, for each $x \in U$ and $w \in W,\|f(x)+w\|<\varepsilon$ and let us remark that, for $x \in U$ and $w \in W, f(x)+w \in B$ if and only if $w=0$. In fact, one of the implications is trivial and, for the other, if $f(x)+w \in B$, then

$$
\delta\|w\| \leq\left\|D g_{0}(w)\right\|=\left\|g(f(x)+w)-g(f(x))-D g_{0}(w)\right\| \leq \frac{\delta}{2}\|w\| ;
$$


hence $w=0$. The derivative at $(0,0)$ of the map $X \times H \rightarrow F,(x, w) \mapsto f(x)+w$, maps $(u, v)$ onto $D f_{0}(u)+v$ and is hence onto, so that a standard result about submersions guarantees the existence of an open set $V$, with $0 \in V \subset Y$, and of smooth maps $\sigma_{1}: V \rightarrow U \subset E$ and $\sigma_{2}: V \rightarrow W \subset H$, satisfying $\sigma_{1}(0)=0$, $\sigma_{2}(0)=0$ and $f\left(\sigma_{1}(y)\right)+\sigma_{2}(y)=y$, for each $y \in V$. By derivation, we have

$$
D f_{0}\left(D \sigma_{10}(v)\right)+D \sigma_{20}(v)=v
$$

hence $D \sigma_{20}$ is the projection from $F$ onto $H$ associated to the direct sum, in particular is onto. As we proved above, for $y \in V$, we have $y \in B$ if, and only if, $\sigma_{2}(y)=0$; hence $B$ is a submanifold of $Y$ at 0 and $T_{0}(B)$ is the kernel of the linear map $D \sigma_{20}: F \rightarrow H$, so that, by (1), $D f_{0}: E \rightarrow T_{0}(B)$ is onto. This implies that $f(X)$ is indeed a neighborhood of 0 in $B$.

Although we will not apply it, we cannot resist stating the following trivial consequence of the previous lemma:

Corollary 1. Let $X$ be a manifold and $f: X \rightarrow X$ be a smooth map such that $f \circ f=f$. Then $f(X)=\{y \in X \mid f(y)=y\}$ is a submanifold of $X$ and

$$
T_{y}(f(X))=D f_{y}\left(T_{y}(X)\right)=\left\{u \in T_{y}(X) \mid D f_{y}(u)=u\right\} .
$$

\section{EMBEDDing A $k$-SYMMETRIC SPACE}

In this section we will fix an integer $k \geq 2$, a Lie group $G$ and a smooth automorphism $\tau: G \rightarrow G$, such that $\tau^{k}=I d_{G}$, and we will consider the corresponding $k$-symmetric space $G / G^{\tau}$, where $G^{\tau}=\{g \in G \mid \tau(g)=g\}$ is the fixed point subgroup. For each $g \in G$, we will denote by $[h]$ the corresponding class in $G / G^{\tau}$.

We will denote $\mathcal{G}=T_{e}(G)$ the Lie algebra of $G$ and $\theta=D \tau_{e}: \mathcal{G} \rightarrow \mathcal{G}$ the corresponding Lie algebra automorphism, that satisfies $\theta^{k}=I d_{\mathcal{G}}$. Of course, the Lie algebra of the subgroup $G^{\tau}$ is $T_{e}\left(G^{\tau}\right)=\mathcal{G}^{\theta}=\{u \in \mathcal{G} \mid \theta(u)=u\}$. The equality

$$
(I d-\theta) \circ\left(I d+\theta+\cdots+\theta^{k-1}\right)=0,
$$

with commuting factors having trivial intersection kernels, implies that $\mathcal{G}=\mathcal{H}_{[e]} \oplus$ $\mathcal{M}_{[e]}$, where

$$
\begin{aligned}
& \mathcal{H}_{[e]}=\mathcal{G}^{\theta}=\operatorname{ker}(I d-\theta)=\left\{u+\theta(u)+\cdots+\theta^{k-1}(u)\right\}_{u \in \mathcal{G}}, \\
& \mathcal{M}_{[e]}=\operatorname{ker}\left(I d+\theta+\cdots+\theta^{k-1}\right)=\{u-\theta(u)\}_{u \in \mathcal{G}} .
\end{aligned}
$$

If $g \in G^{\tau}$, the fact that the conjugation automorphism $c_{g}$ commutes with $\tau$ implies that the Lie algebra automorphism $A d_{g}$ commutes with $\theta$ and hence that the direct sum $\mathcal{G}=\mathcal{H}_{[e]} \oplus \mathcal{M}_{[e]}$ is $A d_{g}$-invariant. We have hence a well-defined structure of reductive homogeneous space on $G / G^{\tau}$, the one that will be considered implicitly. We remark that this is the same reductive structure in $G / G^{\tau}$ that has been defined in [1, using the eigenspaces of the complexification of $\theta$; however the direct approach will be useful later.

Proposition 1. Let us consider the smooth action of $G$ in $G$ defined by $g \cdot h=$ $g h \tau\left(g^{-1}\right)$ and let $\operatorname{Orb}=\left\{g \tau\left(g^{-1}\right)\right\}_{g \in G}$ be the orbit of e for this action. Let $B \subset G$ be the set

$$
B=\left\{h \in G \mid h \tau(h) \cdots \tau^{k-1}(h)=e\right\} .
$$


Then Orb is a submanifold of $G$, open in $B$, and there is an equivariant diffeomorphism $f: G / G^{\tau} \rightarrow$ Orb defined by $f([g])=g \tau\left(g^{-1}\right)$. Moreover, $T_{e}($ Orb $)=\mathcal{M}_{[e]}=$ $\mathcal{M}_{e}$.

Proof. It is straightforward to verify that we have a well defined one-to-one smooth equivariant map $f: G / G^{\tau} \rightarrow G,[g] \mapsto g \tau\left(g^{-1}\right)$, whose image is $\operatorname{Orb}$, so that all we have to prove is that $\operatorname{Orb}$ is a submanifold of $G$, open in $B$. The fact that $B$, like $O r b$, is invariant by the action of $G$ reduces us to proving that $O r b$ is a neighborhood of $e$ in $B$ and that $B$ is a submanifold of $G$ at the point $e$. To simplify notations, let us denote by $\widehat{f}: G \rightarrow G$ the smooth map $g \mapsto g \tau\left(g^{-1}\right)$, whose image is $O r b$. Let $\varphi: G \rightarrow G$ be the smooth map defined by $\varphi(h)=h \tau(h) \cdots \tau^{k-1}(h)$. We have $\varphi(\widehat{f}(g))=e$, for each $g \in G$; in particular $\operatorname{Orb} \subset B$. By differentiating at $e$, we obtain $D \widehat{f}_{e}(u)=u-\theta(u)$ and $D \varphi_{e}(v)=v+\theta(v)+\cdots+\theta^{k-1}(v)$, so that by what was discussed above, both the image of $D \widehat{f}_{e}$ and the kernel of $D \varphi_{e}$ are equal to $\mathcal{M}_{[e]}$. Applying Lemma 1, with a constant map as $\psi$, ends the proof.

The fact that $O r b$ is a reductive homogeneous manifold of the Lie group $G$ gives it a natural $G$-invariant connection. One method of characterizing this connection is to compute the Hessian of the inclusion of $\operatorname{Orb}$ into $G$, when we consider in $G$ its natural symmetric connection. That is what we do now, limiting our computation to what happens at $e \in O r b$, because the general formula can be obtained through the left and right invariance of the connection of $G$. Following the formalism of [2], we compute first the Maurer-Cartan form $\beta_{e}: T_{e}(O r b) \rightarrow \mathcal{M}_{e} \subset \mathcal{G}$. We recall that $\beta_{e}$ is the inverse of the restriction to $\mathcal{M}_{e}$ of the derivative at $e, \rho_{e}: \mathcal{G} \rightarrow T_{e}(\mathrm{Orb})$, of the map $G \rightarrow O r b, g \mapsto g \cdot e=g \tau\left(g^{-1}\right)$, a linear map that is hence defined by $\rho_{e}(u)=u-\theta(u)$.

Lemma 2. The Maurer-Cartan form $\beta_{e}: T_{e}($ Orb $)=\mathcal{M}_{e} \rightarrow \mathcal{M}_{e}$ is defined by

$$
\beta_{e}(v)=-\frac{1}{k} \sum_{j=1}^{k-1} j \theta^{j}(v) .
$$

Proof. All we have to prove is that the linear map $\beta_{e}$, defined above, maps $\mathcal{M}_{e}$ into $\mathcal{M}_{e}$ and verifies $\rho_{e} \circ \beta_{e}=I d_{\mathcal{M}_{e}}$ and this is a straightforward calculation if we recall that $\theta^{k}=I d_{\mathcal{G}}$ and the characterization of $\mathcal{M}_{e}$ as a kernel.

Proposition 2. The Hessian $h_{e}: \mathcal{M}_{e} \times \mathcal{M}_{e} \rightarrow \mathcal{G}$, of the inclusion Orb $\rightarrow G$ at $e$, is given by

$$
h_{e}(u, v)=\left[\beta_{e}(u)-\frac{1}{2} u, v-\theta(v)\right] .
$$

Proof. Let us denote by $\nabla$ and $\nabla^{G}$ the covariant derivatives associated to the connections we are considering in $\operatorname{Orb}$ and in $G$. Let $u, v \in \mathcal{M}_{e}$ and let $Y$ be the vector field on $O r b$ associated to $v$ and to the action of $G$ on $O r b$, that is defined by $Y_{g}=D R_{g_{e}}(v)-D L_{g_{e}}(\theta(v))$, where $R_{g}$ and $L_{g}$ denote the right and left translations by $g$. Let us denote also by $Y$ the vector field on $G$ defined by the same formula. Then $\nabla^{G} Y_{e}(u)=-\frac{1}{2}[u, v+\theta(v)]$ and

$$
\nabla Y_{e}(u)=-\rho_{e}\left(\left[\beta_{e}(u), v\right]\right)=-[u, \theta(v)]-\left[\beta_{e}(u), v-\theta(v)\right],
$$

and the result is now a consequence of the formula $h_{e}(u, v)=\nabla^{G} Y_{e}(u)-\nabla Y_{e}(u)$. 
For $k=2$, we have $\beta_{e}(u)=\frac{1}{2} u$; hence $h_{e}(u, v)=0$, and we retrieve the conclusion that $O r b$ is a totally geodesic submanifold.

Remark 1. There is another equivariant embedding of the $k$-symmetric space $G / G^{\tau}$ that, for $k=2$, coincides with the previous one: The product group $G^{k}$ acts transitively on the manifold $G^{k-1}$ by

$$
\left(g_{1}, \ldots, g_{k}\right) \cdot\left(h_{1}, \ldots, h_{k-1}\right)=\left(g_{1} h_{1} g_{2}^{-1}, g_{2} h_{2} g_{3}^{-1}, \ldots, g_{k-1} h_{k-1} g_{k}^{-1}\right)
$$

and $G^{k-1}$ is then a $k$-symmetric manifold, with the permutation automorphism $\tau: G^{k} \rightarrow G^{k}, \tau\left(g_{1}, \ldots, g_{k}\right)=\left(g_{2}, \ldots, g_{k}, g_{1}\right)$ associated to the base point $(e, \ldots, e)$ $\in G^{k-1}$. The isotropy subgroup is the diagonal $\left\{\left(g_{1}, \ldots, g_{k}\right) \in G^{k} \mid g_{1}=\cdots=g_{k}\right\}$, the corresponding Lie algebra is $\mathcal{H}_{(e, \ldots, e)}=\left\{\left(u_{1}, \ldots, u_{k}\right) \in \mathcal{G}^{k} \mid u_{1}=\cdots=u_{k}\right\}$ and the corresponding direct complement is $\mathcal{M}_{(e, \ldots, e)}=\left\{\left(u_{1}, \ldots, u_{k}\right) \mid u_{1}+\cdots+\right.$ $\left.u_{k}=0\right\}$. We have hence an associated connection on $G^{k-1}$. We can consider the smooth morphism $\psi: G \rightarrow G^{k}, \psi(g)=\left(g, \tau(g), \ldots, \tau^{k-1}(g)\right)$ and we have then a $\psi$-equivariant and $\psi$-reductive map $\Psi: G / G^{\tau} \rightarrow G^{k-1}$,

$$
\Psi([g])=\left(g \tau\left(g^{-1}\right), \tau(g) \tau^{2}\left(g^{-1}\right), \ldots, \tau^{k-2}(g) \tau^{k-1}\left(g^{-1}\right)\right) .
$$

This map is hence totally geodesic and, by looking to the first coordinate, we conclude that it is an embedding of $G / G^{\tau}$ into a submanifold of $G^{k-1}$.

\section{The MANIFOLD $r_{k}(G)$}

In this section we will fix an integer $k \geq 2$ and a Lie group $G$ and we will denote by $r_{k}(G)$ the set of $k$-roots of the identity in $G$,

$$
r_{k}(G)=\left\{g \in G \mid g^{k}=e\right\} .
$$

The group $G$ acts on $r_{k}(G)$ by conjugation: $h \cdot g=c_{h}(g)=h g h^{-1}$. For each $g \in r_{k}(G)$, we will denote by $\operatorname{Orb}_{g}=\left\{h g h^{-1}\right\}_{h \in G}$ the orbit of $g$ for this action. We will denote by $R_{g}$ and $L_{g}$ the right and left translations by $g$.

Proposition 3. The set $r_{k}(G)$ is a closed submanifold of $G$ and the orbits Orb $_{g}$, with $g \in r_{k}(G)$, are open in $r_{k}(G)$. Moreover, for each $g \in r_{k}(G)$,

$$
T_{g}\left(r_{k}(G)\right)=D R_{g_{e}}\left(\mathcal{M}_{g}\right)=D L_{g_{e}}\left(\mathcal{M}_{g}\right)=\left\{D R_{g_{e}}(u)-D L_{g_{e}}(u)\right\}_{u \in \mathcal{G}},
$$

where $\mathcal{M}_{g} \subset \mathcal{G}$ is defined by

$$
\mathcal{M}_{g}=\operatorname{ker}\left(I d+A d_{g}+\cdots+A d_{g}{ }^{k-1}\right)=\left\{u-A d_{g}(u)\right\}_{u \in \mathcal{G}} .
$$

Proof. Let $g \in r_{k}(G)$. Then $c_{g}: G \rightarrow G$ is a smooth automorphism, such that $c_{g}{ }^{k}=I d_{G}$ and the corresponding Lie algebra automorphism is $A d_{g}: \mathcal{G} \rightarrow \mathcal{G}$. By Proposition 1, we conclude that $\operatorname{Orb}_{(g)}=\left\{h c_{g}\left(h^{-1}\right)\right\}_{h \in G}$ is a submanifold of $G$, open in

$$
B_{(g)}=\left\{h \in G \mid h c_{g}(h) \cdots c_{g}^{k-1}(h)=e\right\}
$$

and having $\mathcal{M}_{g}$ as tangent space at $e$. Considering the diffeomorphism $R_{g}: G \rightarrow G$, we conclude that $R_{g}\left(\operatorname{Orb}_{(g)}\right)=O r b_{g}$ is a submanifold of $G$ open in $R_{g}\left(B_{(g)}\right)=$ $r_{k}(G)$ and that $T_{g}\left(r_{k}(G)\right)=T_{g}\left(O r b_{g}\right)=D R_{g_{e}}\left(\mathcal{M}_{g}\right)$. The other characterizations of $T_{g}\left(r_{k}(G)\right)$ follow from the equality $D L_{g_{e}}=D R_{g_{e}} \circ A d_{g}$ and from the $A d_{g}$-invariance of $\mathcal{M}_{g}$. 
Each orbit $\operatorname{Orb}_{g}=\left\{h g h^{-1}\right\}_{h \in G}$ is a homogeneous manifold of $G$ and the restriction of the right translation to the homogeneous manifold $\operatorname{Orb}_{(g)}=\left\{h c_{g}\left(h^{-1}\right)\right\}_{h \in G}$, associated to the automorphism $c_{g}: G \rightarrow G$, is an equivariant diffeomorphism $R_{g}: \operatorname{Orb}_{(g)} \rightarrow \mathrm{Orb}_{g}$. By transport through this equivariant diffeomorphism, $\operatorname{Orb}_{g}$ becomes a reductive homogeneous space, such that, for each $g^{\prime}=h g h^{-1} \in O r b_{g}$, the horizontal space $\mathcal{M}_{g^{\prime}} \subset \mathcal{G}$ is equal to the horizontal space of $\operatorname{Orb}_{(g)}$ at $h c_{g}\left(h^{-1}\right)$; hence

$$
\mathcal{M}_{g^{\prime}}=A d_{h}\left(\mathcal{M}_{e}\right)=\left\{A d_{h}(u)-A d_{h}\left(A d_{g}(u)\right)\right\}_{u \in \mathcal{G}}=\left\{v-A d_{g^{\prime}}(v)\right\}_{v \in \mathcal{G}},
$$

which is compatible with the notation used in the previous proposition and proves, in particular, that the reductive structure in $\mathrm{Orb}_{g}$ does not depend of the choice of $g$ in the orbit.

The reductive homogeneous structure of the submanifolds $\mathrm{Orb}_{g}$ entitles them to, and hence $r_{k}(G)$, with an associated connection. The fact that $R_{g}: \operatorname{Orb}_{(g)} \rightarrow$ $\mathrm{Orb}_{g}$ and $R_{g}: G \rightarrow G$ are totally geodesic maps allows us to deduce the following proposition from Proposition 2:

Proposition 4. For each $g \in r_{k}(G)$, the Hessian $h_{g}: T_{g}\left(r_{k}(G)\right) \times T_{g}\left(r_{k}(G)\right) \rightarrow$ $T_{g}(G)$, of the inclusion $r_{k}(G) \rightarrow G$ at $g$, is defined by

$$
h_{g}\left(D R_{g_{e}}(u), D R_{g_{e}}(v)\right)=D R_{g_{e}}\left(\left[\beta_{(g)}(u)-\frac{1}{2} u, v-A d_{g}(v)\right]\right),
$$

for $u, v \in \mathcal{M}_{g}$, where $\beta_{(g)}(u)=-\frac{1}{k} \sum_{j=1}^{k-1} j A d_{g}{ }^{j}(u)$.

Again, for $k=2$, we have $\beta_{(g)}(u)=\frac{1}{2} u$, hence $h_{g}=0$, and we retrieve the conclusion that $r_{2}(G)$ is a totally geodesic submanifold of $G$.

\section{REFERENCES}

[1] F. E. Burstall: Harmonic Tori in spheres and complex projective spaces. J. reine angew. Math. 469 (1995), 149-177. MR 96m:58053

[2] F. E. Burstall \& J. H. Rawnsley: Twistor Theory for Riemannian Symmetric Spaces. Lecture Notes in Math., vol. 1424, Springer Verlag, 1990. MR 91m:58039

[3] C. Ferreira: Embedding flag manifolds of a Hermitian space $E$ into the unitary group $U(E)$. Boll. Un. Mat. Ital. (7) 7-B (1993), 575-590. MR 94i:58041

[4] C. Ferreira \& A. Machado: Some embeddings of the space of partially complex structures. Portugal. Math. 55 (1998), 485-504. CMP 99:08

[5] A. Gray: Riemannian manifolds with geodesic symmetries of order 3. J. Differential Geom. 7 (1972), 343-369. MR 48:9615

[6] K. Uhlenbeck: Harmonic maps into Lie groups (classical solutions of the chiral model). $J$. Differential Geom. 30 (1989), 1-50. MR 90g:58028

CMaf da Universidade de Lisboa, Av. Prof. Gama Pinto 2, 1649-003 Lisboa, Portugal

E-mail address: cecilia@lmc.fc.ul.pt

E-mail address: armac@lmc.fc.ul.pt 